\title{
Hospital Treatment - Is it AfFordable? A Structured Cost Analysis of Vaginal Deliveries and Planned CAEsarean SECTIONS
}

\author{
I. M. Heer ${ }^{1}$, S. Kahlert ${ }^{2}$, S. Rummel ${ }^{3}$, C. Kümper ${ }^{1}$, W. Jonat ${ }^{1}$, A. Strauss ${ }^{1}$ \\ ${ }^{1}$ Department of Gynecology and Obstetrics, University Hospital of Schleswig-Holstein, Kiel, Germany \\ ${ }^{2}$ Department of Obstetrics and Gynaecology - University of Munich - Grosshadern, Germany \\ ${ }^{3}$ Department of Gynecology and Obstetrics, Klinikum Traunstein, Germany
}

\begin{abstract}
Introduction: The analysis of cost effectiveness in hospitals is as difficult as treating the patients properly. We are yet not able to answer the simple question of what costs are caused by a certain diagnosis and its treatment during an average hospital stay.

Methods: To answer some issues of the global problem of cost effectiveness during hospitalisation, we analysed the costs and the cost structure of a normal obstetrical hospital stay during an uncomplicated vaginal delivery and a planned caesarean section. Cost data was collected and summarized from the patients file, the hospital's computer system gathering all cost centres, known material expenses and expenses of non obstetrical medical services.

Results: For vaginal deliveries/planned caesareans we can calculate with a surplus of about $83 € / 1432 €$. About $45 \%$ of the summarized costs are calculated on a reliable database.

Discussion: The introduction of the DRG based clearing system in Germany has aggravated the discussion on cost effectiveness. Our meticulous work-up of expenses excluded personal precautionary costs and personnel costs of documentation because no tools are described to depict such costs. If we would add these costs to the known expenses of our study, we strongly suspect that hospital treatment of vaginal deliveries or planned caesarean sections is not cost effective.
\end{abstract}

Key words: cost effectiveness, vaginal delivery, caesarean section, DRG

\section{INTRODUCTION:}

Cost effectiveness has become one of the most poplar words in the economic evaluation of medical affairs. The reason is the ongoing discussion of how to make health systems affordable. But the problem of cost effectiveness of medicine is not easily solved because simple basic economic questions are not yet answered properly. For example, the cost of the hospital treatment of a patient over a certain time it is very difficult to name the diversity of expenses (i.e. specialists, nurses, infrastructure). Is the cost of the specialist's man- power more expensive than the infrastructure of the hospital? Basically we are yet not able to answer the simple question of what costs are caused by a certain diagnosis and its treatment during an average hospital stay.

Our study was focused on these problems. To get started with answering some issues of the global problem of cost effectiveness during hospitalisation, we analysed the costs and the cost structure of a normal obstetrical hospital stay during an uncomplicated vaginal delivery and compared our results to a similar analysis of the hospital stay after planned caesarean section. We tried to answer the following questions:

- What are the known individual costs?

- If the hospital is paid by the new system of the German Diagnose Related Groups (G-DRG): Does the corresponding reimbursement cover the related costs of the hospital?

- Can the hospital afford the treatment it offers to the patient?

- Which data of the whole cost analysis can be stated as reliable?

\section{Methods}

Patients: In this retrospective analysis we included only healthy mothers with singleton pregnancies at term. We focused on vaginal deliveries without any obstetrical complication or complicating perinatal diagnosis (i.e. diabetes, high blood pressure, infectious diseases). Similarly to this we elected healthy women with planned caesarean sections conducted at term in epidural anaesthesia without any perioperational complications. The aim of the limitations was to obtain homogenous study populations with a homogenous cost structure to calculate valid average data on the cost structure of the hospital stay.

Type of costs: cost data was collected and summarized from the patients file, the hospital's computer system gathering all cost centres, known material expenses and expenses of non obstetrical medical services.

Our analysis was based on the following definition of cost specifications: 


\section{Costs}

Medical Staff

Non obstetrical medical service institutions

Medical infrastructure

Specification

Doctors

Nurses

\section{Pharmacotherapy}

Wound dressing

Equipment operating theatre

Institute for Anaesthesia

Institute for infectious diseases Blood bank

Laboratory for blood sampling

Pharmacy, sterilization of instruments, disinfections of beds, transport service, medical technical service, hygienic service.

Non medical infrastructure kitchen, laundry, energy, water, cleaning service, administration, central computing services

Most of the costs in the hospital are collected from the central computer system that gathers all data from all different cost centres. Some of these costs are related to a patient's individual case. This allows tracking single costs of the patients hospital stay. We managed our software based cost collection with ISH-SAP ${ }^{\circledR}$, Walldorf, Germany. Some of the individual costs however were though not reconstructable from the patient's records (see below). Here we had to fall back on the centralised computer data.

Calculation of obstetrical personnel costs: We based the personnel cost evaluation on the total costs of the employee for the hospital. These are put together from the negotiated hourly total gross wage rate for employees in the German public health service (2003) and from the employer's contribution.

Medical Staff

Full costs/hour (€)

\begin{tabular}{ll}
\hline Doctors & 46.77 \\
Midwifes and nurses & 31.44
\end{tabular}

All costs caused by the obstetrical medical staff were extracted from the patient's medical record. These were added to costs that are not registered in every case but that are linked to certain occupations that had to be conducted from the obstetrical staff during the patients hospital stay. The linked time consumption and thus the associated costs are calculated after intensive discussion with the whole obstetrical team (i.e. for example time for obstetrical anamnesis 10 minutes, blood sampling 5 minutes, preparation for caesarean by midwife 40 minutes). Both parts of the costs (registered and non registered but defined) were strictly divided to avoid double counting of times and costs.

Calculation of material costs: All costs of medical consumption articles were added if registered in the medical file. These costs were added to the costs of materials that are not registered separately in every single case (i.e. for example sutures, cover sets for the operation theatre, gloves). Both parts (registered and not registered) were strictly divided to avoid double counting of costs.

Calculation of non-obstetrical medical services: During an uncomplicated vaginal delivery or an uncomplicated planned caesarean section the obstetrician needs of medical support from four non-obstetrical institutions:

\section{Anaesthesia}

Epidural anaesthesia was eligible to the patient during vaginal delivery. All Patients with caesarean section had an epidural. The related costs for anaesthesia were extracted from the hospital's computer system. The reason for this kind of data collection (see above) was the inability of the institute for anaesthesia to present own data on their cost structure for an epidural during vaginal delivery or planned caesarean section.

\section{Laboratory for infectious diseases}

As routine, we supply every patient during her first ambulatory visit with a routine test for HIV and Hepatitis C. The related costs of this diagnostic step are billed to the obstetrical clinic.

\section{Laboratory for blood chemistry:}

Every patient (vaginal delivery and planned caesarean) underwent routine blood sampling before vaginal delivery and caesarean section (blood chemistry, blood count and test for coagulation). The related costs were charged to the clinic for obstetrics.

\section{Blood bank:}

All patients underwent tests for their blood group and for blood group antibodies. The related costs were charged to the clinic for obstetrics.

\section{Calculation of costs for medical and non-medical- infrastructure:}

All different costs for medical and non-medical infrastructure are pooled from the associated cost centres that should depict all the above named services. As with the costs of the epidural, we had to rely on the computerized data of the central information system. The problem with the collection of this data is similar as described above. It is the "missing link" between the total costs of the infrastructure services and the associated costs per case.

Returns: All returns paid from the patient's insurance after her discharge from the maternity clinic and data transmission from the hospital are calculated with the German System of Diagnose Related Groups (GDRG). The actual base rate (case weight $=1.0)$ for our institution is calculated with $3200 €$.

Reliability of data: The aim of this study to present data about the costs for defined obstetrical diagnosis is associated with the problem of reliability of such data. We can state that the data from our own documentation of the patient's history are reliable, while the degree of reliability of data that we have extracted from the centralised computer system is less. 


\section{RESULTS}

We analyzed 70 uncomplicated vaginal deliveries and 30 planned caesarean sections of healthy mothers. These patients delivered singletons between July to November 2003 at Munich University Hospital Grosshadern.

Patients: Table 1 shows characteristics of both patient groups (vaginal delivery and planned caesarean section). There is no difference in these key criteria. Thus we can exclude any bias when comparing both groups.

Table 2 shows criteria that differ in the study groups due to the different way of labour.

Costs: All different costs of vaginal delivery and planned caesarean are summarized in Table 3.

Returns: The G-DRG code after uncomplicated vaginal delivery is O60D with a corresponding case weight of 0.57 . The code for an uncomplicated planned caesarean section is $001 \mathrm{D}$ with a corresponding case weight of 1.19. The base rate for case weight 1 is 3200 $€$. Therefore the following returns for the hospital can be assumed:

Procedure

Refund (€)

Uncomplicated vaginal delivery

1820.70

Uncomplicated planned caesarean section

3817.60

Table 4 summarizes costs and refunds thus showing the hospitals profit.

Reliability of data: As stated above, our data of different costs are collected from different sources. Table 5 names and classifies the different sources, thus summarizing the percentage of more and less reliable data of our study. 6 out of 9 cost types can be classified as reliable. However, these 6 cost types represent only $46.22 \%$ (vaginal delivery) and $44.54 \%$ (planned caesarean) of the whole costs.

Table 1. Key criterias for both patient groups.

\begin{tabular}{|c|c|c|c|c|c|}
\hline \multirow[b]{2}{*}{ Age } & \multicolumn{2}{|c|}{ Vaginal delivery } & \multicolumn{2}{|c|}{ Planned caesarean } & \multirow{2}{*}{$\begin{array}{l}\text { Significant difference } \\
\text { No }(p=0.52)\end{array}$} \\
\hline & 30.7 & $(16-43)$ & 31.5 & $(20-38)$ & \\
\hline Gravida & 2 & $(1-5)$ & 2.3 & $(1-6)$ & $\mathrm{No}(\mathrm{p}=0.27)$ \\
\hline Para & 1.7 & $(1-5)$ & 1.9 & $(1-5)$ & $\mathrm{No}(\mathrm{p}=0.47)$ \\
\hline Ambulant visits & 2.5 & $(1-5)$ & 2.8 & $(1-5)$ & $\mathrm{No}(\mathrm{p}=0.28)$ \\
\hline
\end{tabular}

Table 2. Differences in both study groups.

\begin{tabular}{llllll}
\hline & \multicolumn{2}{l}{ Vaginal delivery } & \multicolumn{2}{c}{ Planned caesarean } & Significant difference \\
\hline Pregnancy age at delivery & $39 \mathrm{w} 3 \mathrm{~d}$ & $(38 \mathrm{w} 0 \mathrm{~d}-41 \mathrm{w} 5 \mathrm{~d})$ & $38 \mathrm{w} 2 \mathrm{~d}$ & $(37 \mathrm{w} 0 \mathrm{~d}-40 \mathrm{w} 6 \mathrm{~d})$ & Yes $\left(\mathrm{p}=9.8 \times 10^{-15}\right)$ \\
Birthweight $(\mathrm{g})$ & 3435 & $(2650-4440)$ & 3272 & $(2470-4190)$ & Yes $(\mathrm{p}=0.05)$ \\
Time in delivery room $(\mathbf{H}: \mathbf{M})$ & $10: 44$ & $(2: 00-41.40)$ & $6: 45$ & $(4: 45-10: 50)$ & Yes $\left(\mathrm{p}=5 \times 10^{-4}\right)$ \\
Percentage of peridural & $54 \%$ & $100 \%$ & & & \\
\hline
\end{tabular}

Table 3. Summary total costs vaginal delivery and cesarean section.

\begin{tabular}{llrr}
\hline & & Vaginal delivery (€) & Planned caesarean $(\boldsymbol{€})$ \\
\hline Obstetrical Staff & Doctors & 201.02 & 292.58 \\
& Midwifes & 210.53 & 129.38 \\
Material expenses & Nurses & 144.45 & 316.10 \\
& Materials & 47.69 & 117.86 \\
Non obstetrical medical & Pharmacotherapy & 8.38 & 46.56 \\
service institutions & Anaesthesiology & 51.98 & 375.83 \\
& & & 58.11 \\
& Laboratory for infectious diseases & 47.84 & 13.50 \\
Medical infrastructure & Laboratory for blood chemistry & 6.64 & 88.15 \\
Non medical infrastructure & Blood bank & 73.44 & 137.02 \\
$\sum$ total costs $(\boldsymbol{\epsilon})$ & & 107.68 & 809.81 \\
& & 774.33 & $\mathbf{2 3 8 4 . 9}$ \\
\hline
\end{tabular}


Table 4. Summary of costs and refunds for vaginal delivery and cesarean section.

\begin{tabular}{lrr}
\hline & Vaginal delivery (O60D) & Planned caesarean (O01D) \\
\hline Total costs $(€)$ & -1737.34 & -2384.90 \\
Refunds $(€)$ & +1820.70 & +3817.60 \\
Difference $(€)$ & +83.36 & +1432.70 \\
\hline
\end{tabular}

Table 5. Summary of data reliability.

\begin{tabular}{|c|c|c|c|c|}
\hline \multirow[t]{2}{*}{ Source } & & \multirow[t]{2}{*}{ Reliability given } & \multicolumn{2}{|c|}{ Total stated cost of named source $(€)$} \\
\hline & & & Vaginal Delivery & $\begin{array}{l}\text { Planned caesarean } \\
\text { section }\end{array}$ \\
\hline \multirow[t]{2}{*}{ Patients file } & $\begin{array}{l}\text { Docmented times of } \\
\text { obstetrical staff } 55 \%\end{array}$ & Yes & 305.80 & 405.93 \\
\hline & Documented materials & Yes & 46.49 & 150.35 \\
\hline \multirow[t]{2}{*}{ Assumption } & $\begin{array}{l}\text { Projected times of } \\
\text { obstetrical staff } 45 \%\end{array}$ & Yes & 250.20 & 332.12 \\
\hline & materials & Yes & 9.58 & 14.07 \\
\hline \multirow{3}{*}{$\begin{array}{l}\text { Non obstetrical medical } \\
\text { service institutions }\end{array}$} & Laboratory for infectious diseases & Yes & 47.84 & 58.11 \\
\hline & Laboratory for blood chemistry & Yes & 6.64 & 13.50 \\
\hline & Blood bank & Yes & 73.44 & 88.15 \\
\hline \multirow[t]{3}{*}{ Central computer system } & Anaesthesiology & No & 51.98 & 375.83 \\
\hline & Medical infrastructure & No & 107.68 & 137.02 \\
\hline & Non medical infrastructure & No & 774.33 & 809.81 \\
\hline Total & & & 1737.34 & 2384.89 \\
\hline \multirow[t]{2}{*}{ Summary } & & Safe data & \multicolumn{2}{|c|}{ Safe data of summarized costs $(€)$} \\
\hline & & 6 out of 9 & $\begin{array}{l}803.01 \\
46.22 \%\end{array}$ & $\begin{array}{l}1062.23 \\
44.54 \%\end{array}$ \\
\hline
\end{tabular}

\section{DISCUSSION}

The introduction of the Diagnose Related Group based clearing system in Germany and the still rising costs of the health system have aggraveted the discussion of cost effectiveness in hospitals. What are the costs for a certain procedure? Which parts of the whole system are expensive and where can we save money? The approach to answer these questions is in most cases to initialize external analysis from economic consultants. The authors of this study have experienced such expertises of international consultant firms and have seen the problems of such attempts: Reducing personnel costs unilaterally, not taking into consideration all the other cost factors.

The aim of this study was to answer the following questions: what are our single and total costs? How cost effective are we at treating a defined study group? To answer these questions, we had to determine and summarize every single cost centre and cost factor. Some of these cost factors were easy to define, others were more difficult to evaluate (see Table 3 and 5). In contrast to other studies that focused on cost analysis of defined procedures [1,2] we listed all types of costs separately to classify the reliability of the data. Other studies on cost analysis concerning defined procedures compare the known total costs of the hospital for the defined diagnosis and procedure. These are then divided by the number of patients treated, finally added to the hospitals reimbursement. The result is then interpreted as being cost effective or not $[3,4]$. Other studies just summarize the total charges per episode $[5,6]$. The disadvantage of such calculations is that hidden costs (i.e. infrastructure) often are not collected properly because they are not registered appropriately in cost centres [7]. To our knowledge there is no study in this field that includes the costs of teaching and research of university hospitals. There specific expenses must be much higher than of rural hospitals. This aspect is not considered in the G-DRG system. The result of all these factors is a distortion of expenses.

The severe disadvantage of a software based (in our hospital ISH-SAP ${ }^{\circledR}$, Walldorf, Germany) cost collection is the fact that these data are as accurate as their recording in the different cost centres. Especially the exact depiction of the employee's costs during her/his work is often not possible. The reason for the inefficient allocation of costs is in most cases the fact that doctors are assigned to different units where they produce costs (for example labour ward). Additionally not all assignments are adequately reflected. If they work in a different unit (for example ultrasound), their costs are not depicted because they are officially assigned to the labour ward. On the other hand it is difficult to 
track the exact costs of the same doctor during his occupation with a single case in different cost centres. This is the reason for our attempt to link the different costs (especially the costs for the staff) of a single case with a defined diagnosis as much as possible to the patient's record.

To minimize data dispersion in our study, we focused on simple diagnoses and procedures (vaginal delivery and planned caesarean section) to evolve homogenous data from coherent study groups. We analyzed all cost centres and all sources of different expenses case by case.

The different sources of data and the assignment of costs to a single patient were necessary to complete the cost structure. We had to tolerate a partly inhomogeneous data structure and the assumption that the cost centre based data of the centralised computer system is not as reliable as the data that we collected from the patients file.

To the best of our knowledge, this is the first study that states the percentage of data that can be considered reliable. We were surprised that only $46.22 \%$ (vaginal delivery) and $44.54 \%$ (planned caesarean) of the total costs can be calculated from a reliable database.

Most studies in the field of economic analysis of vaginal and/or caesarean section state that caesarean is linked to higher costs $[5,8]$. On the other hand the refund for caesarean section is higher than for vaginal delivery $[9,10]$. However the range of reported costs for vaginal delivery and/or caesarean section is so wide that we can assume incoherence of the methods used to estimate these costs $[9,11]$.

Despite our meticulous work-up of expenses two cost factors could not be taken into consideration:

1. Personal precautionary costs

2. Personnel costs of documentation (DRG, quality management)

The reason for (1) is the lack of economic methods allowing such calculations for hospitals with the defined profile of care for high-risk patients. The reason for (2) is that the DRG-system is still so new that no reliable information as to the actual documentation effort is possible. A separate evaluation is ongoing to calculate the highly complex structure of personnel costs during the process of documentation. Thus, either cost factors excluded in our cost analysis have to be taken into consideration when judging our cost effectiveness while caring for mothers with vaginal delivery or undergoing planned caesarean section.

The care of our patients is often complicated and relies on various processes. The cost analysis of these structures and processes is complex. Our contribution to this topic is the attempt to list all available costs, thus depicting the structures causing these costs.

Due to rising costs in health care systems, we have to analyze cost structures to understand where we can be more efficient. The established tools to describe these costs are not reliable. This study gives a more detailed view on cost structures in the hospital, thus providing the ongoing financial debate on health care services with more reliable data.
Medical staff nowadays is more conscious about economic needs in the medical field. But there is still little information available during the ongoing workflow about the costs that arise from different medical activities. If electronic patients records would be linked to software based cost analysis, medical staff would have actual reliable information about the generated costs of every case treated.

The rising average age of our patients and the gain of new methods are aligned with higher costs of the health care system. The analysis of cost structures may provide reliable information about possible and required cost reductions. The available tools to do so are still not sufficient. Further prospective studies have to establish similar methods to generate more reliable data about costs and cost structures.

\section{REFERENCES}

1. Mishra, V., et al., A comparison of actual registered costs and costs derived from diagnosis-related groups (DRGs) for patients undergoing heart transplantation, lung transplantation, and thoracotomy for other lung diseases. Transpl Int, 2001. 14(6): p. 361-9.

2. Skeie, B., et al., A comparison of actual cost, DRG-based cost, and hospital reimbursement for liver transplant $\mathrm{pa}-$ tients. Transpl Int, 2002. 15(9-10): p. 439-45. Epub 2002 Sep 24.

3. Mathisen, A.B., et al., [High-risk pregnancy, costs and DRG reimbursement]. Tidsskr Nor Laegeforen, 2002. 122(5): p. 503-6.

4. Bost, B.W., Cesarean delivery on demand: what will it cost? Am J Obstet Gynecol, 2003. 188(6): p. 1418-21; discussion 1421-3.

5. Allen, V.M., et al., Economic implications of method of delivery. American Journal of Obstetrics and Gynecology, 2005. 193(1): p. 192-197.

6. Vahé A. Kazandjian, C.P.C.S.O.K.W., Does a Cesarean section delivery always cost more than a vaginal delivery? 2007. p. 16-20.

7. Clark, L., M. Mugford, and C. Paterson, How does the mode of delivery affect the cost of maternity care? $\mathrm{Br} \mathrm{J}$ Obstet Gynaecol, 1991. 98(6): p. 519-23.

8. Susan R. Miesnik, B.J.R., A Review of Issues Surrounding Medically Elective Cesarean Delivery. 2007. p. 605-615.

9. Malkin, J.D., Not As Much As You Think: Toward a Truer Estimate of the Difference in Direct Medical Costs Between Vaginal and Cesarean Deliveries. 2001. p. 208209.

10. Wagner, M., Choosing caesarean section. The Lancet, 2000. 356(9242): p. 1677-1680.

11. Zupancic, J.A.F., The Economics of Elective Cesarean Section. Clinics in Perinatology, 2008. 35(3): p. 591-599.

Received: January 14, 2009 / Accepted: June 8, 2009

Address for correspondence:

Dr. Ivo Markus Heer

Klinik und Poliklinik für Frauenheilkunde und Geburtshilfe und Michaelis-Hebammenschule

des Universitätsklinikums Schleswig-Holstein - Campus Kiel

Michaelisstraße 16

24105 Kiel

Germany

Phone: $\quad++494315972100$

Fax: $\quad++494315972110$

Email: iheer@email.uni-kiel.de 